Check for updates

Cite this: Chem. Sci., 2019, 10, 3976

๑ All publication charges for this article have been paid for by the Royal Society of Chemistry

Received 26th December 2018 Accepted 25th February 2019

DOI: $10.1039 / \mathrm{c} 8 \mathrm{sc} 05771 \mathrm{k}$

rsc.li/chemical-science

\section{A cationic polymethacrylate-copolymer acts as an agonist for $\beta$-amyloid and an antagonist for amylin fibrillation $\uparrow$}

\author{
Bikash R. Sahoo, (D) a Takuya Genjo, ${ }^{a}$ Takahiro W. Nakayama, ${ }^{\text {b }}$ Andrea K. Stoddard, ${ }^{a}$ \\ Toshio Ando, (D) ${ }^{\mathrm{b}}$ Kazuma Yasuhara, ${ }^{\mathrm{C}}$ Carol A. Fierke ${ }^{\text {ad }}$ \\ and Ayyalusamy Ramamoorthy (iD *a
}

In humans, $\beta$-amyloid and islet amyloid polypeptide (IAPP, also known as amylin) aggregations are linked to Alzheimer's disease and type-2 diabetes, respectively. There is significant interest in better understanding the aggregation process by using chemical tools. Here, we show the ability of a cationic polymethacrylate-copolymer (PMAQA) to quickly induce a $\beta$-hairpin structure and accelerate the formation of amorphous aggregates of $\beta$-amyloid-1-40, whereas it constrains the conformational plasticity of amylin for several days and slows down its aggregation at substoichiometric polymer concentrations. NMR experiments and microsecond scale atomistic molecular dynamics simulations reveal that PMAQA interacts with $\beta$-amyloid-1-40 residues spanning regions K16-V24 and A30-V40 followed by $\beta$-sheet induction. For amylin, it binds strongly close to the amyloid core domain (NFGAIL) and restrains its structural rearrangement. High-speed atomic force microscopy and transmission electron microscopy experiments show that PMAQA blocks the nucleation and fibrillation of amylin, whereas it induces the formation of amorphous aggregates of $\beta$-amyloid-1-40. Thus, the reported study provides a valuable approach to develop polymer-based amyloid inhibitors to suppress the formation of toxic intermediates of $\beta$-amyloid-1-40 and amylin.

\section{Introduction}

Self-assembly of amyloidogenic proteins is involved in numerous neurodegenerative diseases and other diseases including Alzheimer's disease (AD) and type-2 diabetes (T2D). ${ }^{\mathbf{1 , 2}}$ Nevertheless, our current understanding of the role of protein aggregation in the pathogenesis of such diseases remains elusive. ${ }^{3}$ Despite the recent advancements in high-throughput screening of several anti-amyloidogenic compounds, ${ }^{4}$ there is no treatment for protein aggregation based disorders including $\mathrm{AD}$ and T2D. ${ }^{5} \beta$-amyloid and islet amyloid polypeptide (amylin) aggregations are linked to $\mathrm{AD}$ and T2D, respectively. ${ }^{6}$ Both $\beta$ amyloid and amylin follow a conserved aggregation pathway and form toxic oligomer intermediates. Thus, they have been the subject of intense research to establish a pathological correlation and develop small molecules that interfere with the

\footnotetext{
aBiophysics and Department of Chemistry, University of Michigan, Ann Arbor, MI 48109-1055, USA. E-mail: ramamoor@umich.edu

${ }^{b}$ Bio-AFM Frontier Research Center, Kanazawa University, Kanazawa 920-1192, Japan ${ }^{c}$ Graduate School of Materials Science, Nara Institute of Science and Technology, Ikoma, Nara 6300192, Japan

${ }^{d}$ Department of Chemistry, Texas A\&M University, College Station, TX 77843, USA

$\dagger$ Electronic supplementary information (ESI) available. See DOI: $10.1039 / \mathrm{c} 8 \mathrm{sc} 05771 \mathrm{k}$
}

aggregation pathways of $\beta$-amyloid and amylin. ${ }^{6}$ The sequential protein aggregation mechanism from a water-soluble monomer to insoluble amyloid fibers triggering $\mathrm{AD}$ and $\mathrm{T} 2 \mathrm{D}$ still remains elusive. However, several studies have observed a conserved pathway where both $\beta$-amyloid and amylin monomers aggregate to form pre-fibrillar toxic oligomers followed by matured $\beta$ sheet rich fiber structures. ${ }^{7}$ Thus, the sequential aggregation products of $\beta$-amyloid or amylin have been targeted to design potential inhibitors to interrupt amyloid formation., ${ }^{\mathbf{4} 8}$ Moreover, substantial research effort has been devoted to developing strategies to reduce the formation of toxic intermediates of $\beta$ amyloid or amylin. In response to this, several amyloid inhibitors or modulators have been clinically tested for AD or T2D treatment. However, small molecule inhibitors or modulators targeting amyloidosis have recently faced several clinical trial challenges.8,9 In addition, the complexities associated with amyloid aggregation such as biological pathways, involvement of other cellular key players, structural heterogeneity, etc., pose challenges to current therapeutic developments. Among several compounds, chemically conserved scaffold molecules characterized by multi-aromatic groups have been tested in vitro or in vivo. ${ }^{10-14}$ These compounds have been reported to modulate the amyloid aggregation pathways. But the poor solubility and bioavailability affect their therapeutic nature and recently have been shown to be overcome using nanocarriers. ${ }^{15-17}$ Although, 
their mechanism of action remains unknown, covalent bonding, hydrogen bonding, hydrophobic interactions and $\pi-\pi$ stacking are thought to be the major driving forces for their inhibitory mechanism of action. ${ }^{18-21}$ Oxidation of amyloid peptides by chemical compounds can also modulate amyloid aggregation. ${ }^{22}$ Despite substantial efforts, no significant new drugs have been discovered against these most tenacious and unnerving medical disorders. The successive failures of small molecule compounds direct researchers to develop new antiamyloidogenic molecules such as polymers, peptoids, macrocyclic peptides, nanoparticles, molecular chaperones, etc. ${ }^{\mathbf{8} 23-27}$ Among them, several polymers characterized by their ionic properties have been tested to investigate their activities towards amyloidogenic aggregation. Notably, amine containing polymers, polyamino acids, cationic surfactants and cellular polyamines have been observed to modulate $\beta$-amyloid aggregation. ${ }^{28-30}$ Similarly, controlled aggregation kinetics and toxicity of amylin using star-polymers and $\beta$-amyloid using polymer-nanodiscs have been studied recently. ${ }^{19,20,25}$ Here we demonstrate the modulation of amyloid aggregation pathways for amylin and $\beta$-amyloid-1-40 using a polymethacrylate derived copolymer (PMAQA) that has been implicated in several biological studies including lipid-nanodisc formation, enhancement of drug delivery, modulation of bioavailability and microencapsulation. ${ }^{31-33}$

\section{Experimental section}

\section{Materials}

The polymethacrylate quaternary ammonium copolymer (PMAQA, $\sim 4.7 \mathrm{kDa}$ ) was synthesized and purified as reported elsewhere. ${ }^{31}$ Unlabeled and uniform ${ }^{15} \mathrm{~N}$ isotope labeled full-length $\beta$-amyloid-1-40 (DAEFRHDSGYEVHHQKLVFFAEDVGSNKGAIIGLMVGGVV) was recombinantly expressed in $E$. coli BL21 (DE3). The $\beta$-amyloid1-40 expression followed previously described protocols ${ }^{\mathbf{3 4 , 3 5}}$ and purified by loading the samples onto an ${ }^{\text {ECO }}$ PLUS HPLC column packed with the reversed-phase separation material. Synthetic human amylin (KCNTATCATQRLANFLVHSSNNFGAILSSTNVGSNTY- $\mathrm{NH}_{2}$ ) was purchased from AnaSpec at $>95 \%$ purity.

\section{Sample preparation}

The sample preparation followed a previously described method. ${ }^{27}$ Briefly, $1 \mathrm{mg} \mathrm{mL}{ }^{-1} \beta$-amyloid-1-40 peptide was dissolved in $5 \%(\mathrm{v} / \mathrm{v}) \mathrm{NH}_{4} \mathrm{OH}$ and aliquoted to $0.1 \mathrm{mg} \mathrm{mL}^{-1}$ followed by lyophilization. The $\beta$-amyloid-1-40 peptide powder was re-suspended in $10 \mathrm{mM}$ sodium phosphate, $\mathrm{pH} 7.4$, and sonicated for $30 \mathrm{~s}$ followed by centrifugation at $14000 \times \mathrm{g}$ for $15 \mathrm{~min}$ at $4{ }^{\circ} \mathrm{C}$ to remove small aggregates. $1 \mathrm{mg} \mathrm{mL}{ }^{-1}$ amylin was treated with 1,1,1,3,3,3-hexafluoroisopropanol (HFIP) and kept on ice for 30 minutes. The peptide solutions were aliquoted to $0.1 \mathrm{mg} \mathrm{mL} \mathrm{m}^{-1}$ and lyophilized. The amylin powder was resuspended in $30 \mathrm{mM}$ sodium acetate buffer, $\mathrm{pH}$ 5.5, and sonicated for $30 \mathrm{~s}$. The PMAQA powder $\left(10 \mathrm{mg} \mathrm{mL}^{-1}\right)$ was dissolved in deionized water.

\section{Thioflavin-T fluorescence assay}

Thioflavin $\mathrm{T}$ (ThT) fluorescence assays were performed to monitor the aggregation kinetics of $5 \mu \mathrm{M} \beta$-amyloid-1-40 or amylin at $37^{\circ} \mathrm{C}$ in the presence of PMAQA $(0.1,0.2,1,2.5,5,6$, 7.5, 10, and $50 \mu \mathrm{M}$ ) and $10 \mu \mathrm{M}$ ThT. Fisher 96-well polystyrene plates with a sample volume of $100 \mu \mathrm{L}$ per well in triplicate were used for the ThT assay. The kinetics of amyloid formation was monitored at 3 min intervals under no-shaking conditions for 4 days using a microplate reader (Biotek Synergy 2) with excitation and emission wavelengths of 440 and $485 \mathrm{~nm}$, respectively.

\section{Circular dichroism and Fourier transform-infrared spectroscopy}

$\beta$-amyloid-1-40 or amylin secondary structural transition in the presence or absence of PMAQA was studied by Far-UV circular dichroism (CD) using a JASCO (J820) spectropolarimeter. A $1 \mathrm{~mm}$ light-path length cuvette containing $25 \mu \mathrm{M} \beta$-amyloid-140 or amylin solution titrated with an increasing concentration of PMAQA (ranging from 0.25 to $50 \mu \mathrm{M}$ ) was used to monitor the evolution of structural transition at $25{ }^{\circ} \mathrm{C}$. The samples (peptide : polymer $=1: 1.5$ ) were stored at room temperature and the CD spectra were recorded for 5 days at different time intervals. The CD spectra were averaged and conveyed as the mean residue ellipticity $[\Theta]$ after subtracting the signal from a solution without peptide. Fourier transforminfrared (FT-IR) spectra were measured for $25 \mu \mathrm{M} \beta$-amyloid-140 or amylin mixed with 1.5 molar equivalent of PMAQA (incubated for 6 hours followed by lyophilization) in transmission mode within a range of 4000-400 $\mathrm{cm}^{-1}$ using a Thermos scientific ATR-FTIR instrument. $10 \mu \mathrm{M}$ of $\beta$-amyloid1-40 or amylin was incubated with $15 \mu \mathrm{M}$ of PMAQA for $\sim 5$ minutes and overnight, respectively, for size distribution analysis. The resultant solutions were purified using size exclusion chromatography (SEC) by passing through a Superdex 200 Increase 300/10 GL column operated on an AKTA purifier (GE Healthcare, Freiburg, Germany).

\section{NMR}

1D and 2D NMR spectra were recorded on a $600 \mathrm{MHz}$ Bruker Avance III NMR spectrometer equipped with a $z$-axis gradient cryogenic probe. Unlabeled amylin $(50 \mu \mathrm{M})$ dissolved in $30 \mathrm{mM}$ sodium acetate, $\mathrm{pH}$ 5.5, and unlabeled $\beta$-amyloid-1-40 or uniformly ${ }^{15} \mathrm{~N}$-labeled $\beta$-amyloid-1-40 peptides $(60 \mu \mathrm{M})$ dissolved in $10 \mathrm{mM}$ sodium phosphate, $\mathrm{pH} 7.4$, and buffer containing $90 \% \mathrm{H}_{2} \mathrm{O} / 10 \%{ }^{2} \mathrm{H}_{2} \mathrm{O}$ were used for NMR measurements. The $2 \mathrm{D}^{15} \mathrm{~N} /{ }^{1} \mathrm{H}$ SOFAST-HMQC ${ }^{36}$ NMR titration experiments of $\beta$-amyloid-1-40 $(60 \mu \mathrm{M})$ with 0.6, 1.2 and $3 \mu \mathrm{M}$ PMAQA were performed at $10{ }^{\circ} \mathrm{C}$ with 64 scans and $200 \mathrm{t} 1$ increments. The NMR spectra were processed using TopSpin 3.5 (Bruker) and analyzed using Sparky. ${ }^{37}$

\section{High-speed AFM and transmission electron microscopy imaging}

A high-speed atomic force microscopy (HS-AFM) instrument ${ }^{38,39}$ was operated in the tapping mode with a small cantilever (BL- 
AC10DS-A2, Olympus with spring constant $k=0.1 \mathrm{~N} \mathrm{~m}^{-1}$, and resonance frequency $f=\sim 400 \mathrm{kHz}$ in water). Each cantilever has an amorphous carbon tip at its top. The tip was prepared by electron beam deposition with a scanning electron microscope (ELS-7500, Elionix). The free oscillation amplitude of the cantilever and the typical set-point were $\sim 1 \mathrm{~nm}$ and $80 \%$ of the free oscillation amplitude. For the HS-AFM sample stage, a freshly cleaved mica disc (of $1 \mathrm{~mm}$ in diameter) fixed on a glass rod ( $2 \mathrm{~mm}$ in diameter) was used. Before the deposition of amylin fibril seeds, each mica disc was freshly cleaved. For 3aminopropyltriethoxysilane (APTES)-modification, mica was immersed in $2 \mu \mathrm{L}$ of ten-thousand-fold dilution of APTES with water followed by the removal of unbound APTES with $60 \mu \mathrm{L}$ of water. Amylin fibril seeds for the imaging were prepared by incubation of $60-100 \mu \mathrm{L}$ of $5 \mu \mathrm{M}$ amylin in $30 \mathrm{mM}$ sodium acetate, $\mathrm{pH} 5.5$, for 2-3 days followed by sonication with a handheld sonicator (UR-21P, TOMY). The validation of the sonication conditions was performed using AFM images (typical sonication conditions: ten times of $\sim 0.5 \mathrm{~s}$ at the output level 3). $2 \mu \mathrm{L}$ of fibril seeds was deposited on bare mica or APTES-modified mica followed by incubation for about 5 minutes. After the removal of unbound seeds with $20 \mu \mathrm{L}$ of $30 \mathrm{mM}$ sodium acetate, $\mathrm{pH}$ 5.5, the scanner with the sample stage was set on the scanner holder so that the stage was immersed in $60 \mu \mathrm{L}$ of $30 \mathrm{mM}$ sodium acetate, $\mathrm{pH} 5.5$, in the sample chamber with the cantilever. After the observation of the fibril seeds, the buffer solution in the chamber was replaced with $5 \mu \mathrm{M}$ amylin with/without equimolar PMAQA. HS-AFM observation was started just after the replacement of the sample chamber solution. The HS-AFM movie analysis and kymographs were prepared using the ImageJ (NIH) plugin as described elsewhere. ${ }^{40}$

$5 \mu \mathrm{M}$ of $\beta$-amyloid-1-40 monomers dissolved in $10 \mathrm{mM}$ sodium phosphate, $\mathrm{pH} 7.4$, or $5 \mu \mathrm{M}$ of amylin monomers dissolved in $30 \mathrm{mM}$ sodium acetate, $\mathrm{pH}$ 5.5, were incubated for 24 hours under continuous agitation with/without an equimolar concentration of PMAQA. $\beta$-amyloid-1-40 or amylin fibers were prepared by incubating $5 \mu \mathrm{M}$ of peptide monomers in respective buffers as described above at room temperature for 3 days under continuous agitation. Then, the fibers were sonicated using an ultrasonic bath sonicator for 2 hours at room temperature to generate short fibers, which were used as amyloid seeds. The sonicated fibers were incubated with $5 \mu \mathrm{M}$ of PMAQA in respective peptide buffers at room temperature for 24 hours under continuous agitation to check the effect of the polymer on the fiber morphology. In addition, to monitor the effect of PMAQA on the self-seeding reaction, sample solutions containing sonicated fibers were incubated with 5 $\mu \mathrm{M}$ monomers of respective peptides for 24 hours in the presence or absence of $5 \mu \mathrm{M}$ PMAQA. $10 \mu \mathrm{L}$ of all sample solutions described above were added to a collodion-coated copper grid and incubated for 3 minutes at room temperature followed by three times rinsing with double deionized water. Then, the copper grid was stained with $3 \mu \mathrm{L}$ of $2 \%(\mathrm{w} / \mathrm{v})$ uranyl acetate and incubated for 2 minutes followed by three times rinsing with double deionized water. The sample grids were dried overnight under vacuum and used to obtain transmission electron microscopy (TEM) images using a HITACHI H-7650 transmission microscope (Hitachi, Tokyo, Japan) at $25^{\circ} \mathrm{C}$.

\section{MD simulations}

The 2D structure of PMAQA (Fig. S1†) was created using ChemDraw 16.0 and exported to Chem3D for energy minimization. The 3D structure of PMAQA and its topology files were created using ATB builder ${ }^{\mathbf{4 1}}$ from the energy minimized 2D structure for all-atom molecular dynamics (MD) simulation. The solution NMR structures of $\beta$-amyloid-1-40 (PDB ID: 2LFM ${ }^{42}$ and amylin (PDB ID: $\left.5 \mathrm{MGQ}\right)^{43}$ were considered as the initial structures for PMAQA interaction analysis; the amino acid sequences of the peptides are shown in Fig. S1. $\uparrow$ The MD system was built in the GROMACS ${ }^{44}$ software package, version 5.0.7 (GROMOS96 54A7 force field ${ }^{45}$ ), by placing $\beta$-amyloid-1-40 or amylin at the center of a cubic box and the polymer $\sim 1 \mathrm{~nm}$ away from the protein. The MD systems were solvated using SPC/E water $\left(\approx 1000 \mathrm{~kg} \mathrm{~m}^{-3}\right)$ and neutralized by adding counter ions followed by energy-minimization using the steepestdescent method. Short NVT (100 ps) followed by 1 ns NPT ( $310 \mathrm{~K}$ and 1 bar) was performed to equilibrate the MD systems. MD simulations were carried out using 3D periodic boundary conditions over a production run of 0.7 and $1 \mu$ s for amylinPMAQA and $\beta$-amyloid-PMAQA systems, respectively. MD trajectories were interpreted using visual molecular dynamics ${ }^{\mathbf{4 6}}$ and images were built using Discovery studio visualizer 3.5. The binding free energy was calculated for amylin-PMAQA and $\beta$ amyloid-PMAQA complexes using MM/PBSA by retrieving 500 structures from the last $100 \mathrm{~ns}$ MD simulation. ${ }^{47}$ The GMXAPBS tool was used for the free energy calculation as described elsewhere. ${ }^{48-50}$

\section{Results and discussion}

The effect of PMAQA on $\beta$-amyloid-1-40 and amylin aggregation kinetics was monitored using ThT based fluorescence assay. ThT aggregation assays performed for 4 days showed opposite
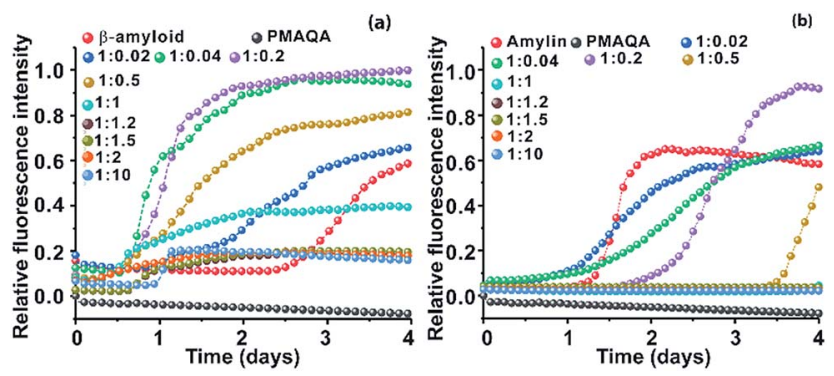

Fig. 1 Effect of PMAQA on the aggregation kinetics of $\beta$-amyloid-140 or amylin. Relative ThT fluorescence of $5 \mu \mathrm{M} \beta$-amyloid-1-40 in $10 \mathrm{mM}$ sodium phosphate, $\mathrm{pH}$ 7.4, (a) or amylin in $30 \mathrm{mM}$ sodium acetate, $\mathrm{pH} 5.5$, (b) in the presence of variable PMAQA concentrations at the indicated peptide to polymer molar ratios. The data in both graphs represent the average of ThT values performed in triplicate as shown in Fig. S2.† 
activities for $\beta$-amyloid-1-40 and amylin aggregation in the presence of PMAQA (Fig. 1 and S2 $\dagger$ ). As shown in Fig. $1 \mathrm{a}$ and b, PMAQA accelerated $\beta$-amyloid-1-40 aggregation and slowed down amylin aggregation. At a low polymer concentration, i.e. a peptide : PMAQA molar ratio of $1: 0.02$ or $1: 0.04$, a significant difference in the lag-times of $\beta$-amyloid-1-40 and amylin aggregation was ascertained (Fig. 1a and b). In the presence of 0.02 molar equivalent of PMAQA, the $\beta$-amyloid-1-40 fibrillation growth was observed after two days (blue) as compared to the aggregation after three days for the control sample (red). A slight increase in the polymer concentration, i.e. 0.04 molar equivalent of PMAQA (green), significantly accelerated $\beta$ amyloid-1-40 fibrillation with an increased ThT fluorescence intensity (Fig. 1a and S2a $\dagger$ ), while a further increase in the polymer concentration to a $1: 0.2$ or $1: 0.5 \beta$-amyloid : PMAQA molar ratio also accelerated the peptide aggregation. But, a relatively lower ThT intensity was observed for $1: 0.5$ as compared to that obtained for $1: 0.2$ (or $1: 0.04$ ). As shown in Fig. 1a, the observed ThT intensities are higher for all the substoichiometric concentrations of PMAQA than the control (i.e., in the absence of PMAQA). At an equimolar or superstoichiometric concentration of PMAQA (Fig. 1a and S2b $\dagger$ ), $\beta$ amyloid-1-40 fibrillation was $\approx 6$ times faster than that observed for the control sample.

Unlike the effect of PMAQA on $\beta$-amyloid-1-40 fibrillation, amylin aggregation was slowed down at substoichiometric PMAQA concentrations, i.e. an amylin : PMAQA molar ratio of $1: 0.02$ or $1: 0.04$ (Fig. S2c $\dagger$ ). Increasing the polymer concentration to a $1: 0.2$ amylin : PMAQA molar ratio further slowed down the aggregation with a lag-time of over 2 days as compared to its aggregation in $\sim 1$ day in the absence of PMAQA (Fig. 1b). A further increase in PMAQA concentration, i.e. at equimolar polymer : peptide or superstoichiometric PMAQA concentrations, amylin aggregation was significantly slowed down as revealed by the negligibly small $\mathrm{ThT}$ fluorescence intensity (Fig. $1 \mathrm{~b}$ and S2d $\dagger$ ). The observed increase in the ThT intensity in both peptides at substoichiometric PMAQA concentrations (peptide : polymer 1:0.5 or lower) indicates the presence of a large number of $\beta$-amyloid or amylin fibers. On the other hand, at superstoichiometric PMAQA concentrations (peptide : polymer $1: 1$ or higher), a substantial decrease in the ThT intensity indicates the presence of a smaller number of fibers. Specifically, the low ThT intensity observed for $\beta$-amyloid-1-40 could also indicate the formation of morphologically distinct fibers, i.e. amorphous like aggregates that depict low ThT fluorescence intensity. ${ }^{51}$

Next, the conformational changes in both peptides in the presence and absence of PMAQA were investigated using far-UV $\mathrm{CD}$ experiments. The CD spectra revealed a gradual structural transition (from unfolded to folded) in $\beta$-amyloid-1-40 when titrated with a $1: 0.01$ or $1: 0.04 \beta$-amyloid : PMAQA molar ratio as indicated by a reduction in CD molar ellipticity $[\Theta]$ and a small change in $\mathrm{CD}$ minima at $\approx 200 \mathrm{~nm}$ (Fig. 2a). The reduction in $[\Theta]$ indicates $\beta$-amyloid-1-40 aggregation and is in line with the observed ThT aggregation (Fig. 1a). A partial helical CD spectrum containing $\alpha / \beta$ secondary contents of $9.4 /$ $26.1 \%$ (as estimated by BESTSEL ${ }^{52}$ ) was observed at a $1: 0.2 \beta$ -
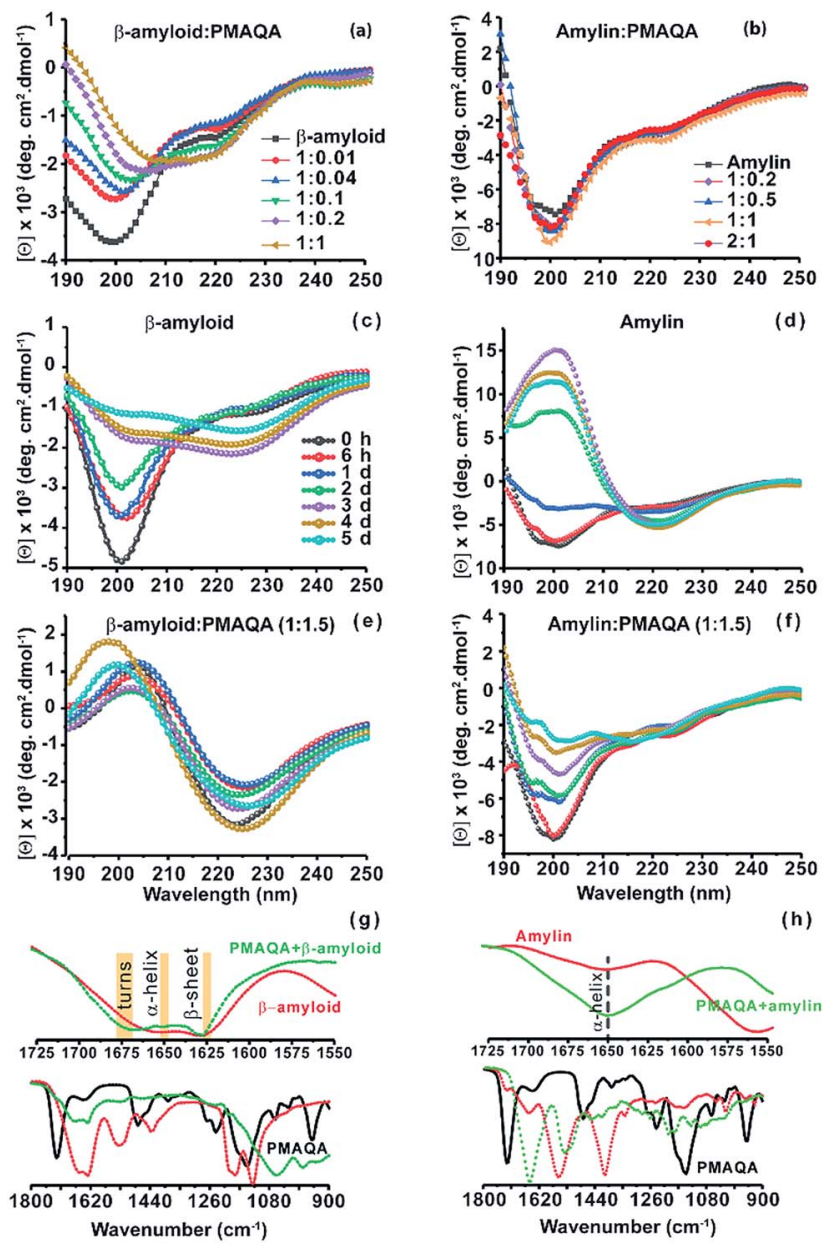

Fig. 2 Conformational analysis of $\beta$-amyloid-1-40 and amylin with and without PMAQA. Far-UV CD measurements showing the structural changes in $25 \mu \mathrm{M}$ of $\beta$-amyloid-1-40 in $10 \mathrm{mM}$ sodium phosphate, $\mathrm{pH} 7.4$, (a) and in $25 \mu \mathrm{M}$ of amylin in $30 \mathrm{mM}$ sodium acetate, $\mathrm{pH}$ 5.5 , (b) in the presence of PMAQA incubated for $\sim 10$ minutes at room temperature at the indicated peptide: PMAQA molar ratios ( $a$ and $b$ ). Time-lapsed structural changes in 25 $\mu \mathrm{M} \beta$-amyloid-1-40 (c and e) and amylin ( $d$ and $f$ ) in the absence ( $c$ and d) and presence (e and f) of 1.5 molar equivalent PMAQA; time intervals used for results presented in $\mathrm{c}-\mathrm{f}$ are indicated in (c). FT-IR spectra of $\beta$-amyloid-1-40 (g) and amylin (h) in the presence (green) and absence (red) of PMAQA obtained after 6 hours; peptide concentration and buffer conditions were as described for the samples used in CD measurements. Changes in the secondary structure of $\beta$-amyloid-1-40 are highlighted in yellow in the fingerprint regions $\left(1550-1725 \mathrm{~cm}^{-1}\right.$ ) (g, top).

amyloid : PMAQA molar ratio (Fig. 2a). A further increase in PMAQA concentration to a $1: 1 \beta$-amyloid : PMAQA molar ratio marginally increased the $\beta$-sheet contents in $\beta$-amyloid-1-40 $(\alpha /$ $\beta$ content of $7.7 / 28.2 \%$ ). The shift of $\mathrm{CD}$ minima at $\approx 200 \mathrm{~nm}$ and decrease in $[\Theta]$ with an increasing concentration of PMAQA indicated its activity in accelerating $\beta$-amyloid-1-40 aggregation as observed in the ThT assay (Fig. 1a). Remarkably, unlike $\beta$ amyloid-1-40, no significant conformational changes were observed in amylin when titrated with 0.2 to 2 equivalents of PMAQA with a CD minimum centered at $\approx 200 \mathrm{~nm}$ with a very 
little change in $[\Theta]$ that represents a partially folded randomcoil rich conformation (Fig. 2b).

We next monitored the stability of the PMAQA induced $\beta$ amyloid-1-40 or amylin secondary structures for 5 days by incubating the peptides at room temperature in the absence or presence of 1.5 molar equivalents of PMAQA (Fig. 2c-f). The time-lapse $C D$ spectra of $\beta$-amyloid-1-40 or amylin in the absence of PMAQA showed a sequential structural transition from a random-coil rich (negative peak $\approx 200 \mathrm{~nm}$ ) to $\beta$-sheet structure (Fig. 2c and d). While $\beta$-amyloid-1-40 in solution is reported to exhibit a sequential structural change to form cross $\beta$-sheet structures over several days (Fig. $2 \mathrm{c}$ ), ${ }^{53}$ in the presence of 1.5 molar equivalents of PMAQA a rapid $\beta$-sheet induction within several minutes was observed (Fig. 2e). The observed rapid structural change correlates with the ThT aggregation kinetics (Fig. 1a) and indicates a reduction in the level of potential $\beta$-amyloid-1-40 oligomers that are reported to be neurotoxic. ${ }^{53,54}$

Similar to the amyloidogenic properties of $\beta$-amyloid-1-40, amylin in solution after 2 days depicted a CD spectrum with a maximum at $\approx 200$ and a minimum at $\approx 218 \mathrm{~nm}$ indicating its transitory state characterized by an increasing percentage of $\beta$ sheets (54\%) (Fig. 2d). But, unlike $\beta$-amyloid-1-40, and as observed in ThT assays for amylin (Fig. 1b), CD spectra showed a relatively slow change in the secondary structure of amylin when incubated with a 1.5 molar excess of PMAQA. The CD minimum was observed at $\approx 200 \mathrm{~nm}$ up to day 3 and the spectra were quantified using BESTSEL (Fig. $2 \mathrm{f}$ and Table 1). Secondary structure assessment of PMAQA bound amylin from CD spectra showed a relative increase in $\alpha$-helix (14.5\%) and decrease in $\beta$ sheet $(21.7 \%)$ content as compared to that observed in the absence of PMAQA (Table 1). In addition, an increased percentage of parallel $\beta$-sheets ( $13.4 \%$ on day 5 ) was observed for amylin in the presence of PMAQA over time as compared to $0 \%$ (on day 5) in the absence of PMAQA. This observation indicates that PMAQA bound amylin could have both antiparallel and parallel $\beta$-structures. Such observations have been found previously using X-ray crystallography in different segments of amylin (segments 13-18 with parallel and antiparallel $\beta$-structures for segments $16-21,22-28$, and 23-29). ${ }^{55}$ On the other hand, $\beta$-amyloid-1-40 showing a positive CD band

Table 1 Secondary structure assessment (\%) of amylin from CD spectra (Fig. 2d and f) in the absence or presence of PMAQA by BESTSEL ${ }^{52}$

\begin{tabular}{|c|c|c|c|c|c|c|}
\hline Time & $0 \mathrm{~d}$ & $1 \mathrm{~d}$ & $2 \mathrm{~d}$ & $3 \mathrm{~d}$ & $4 \mathrm{~d}$ & $5 \mathrm{~d}$ \\
\hline \multicolumn{7}{|c|}{ Amylin in $30 \mathrm{mM}$ sodium acetate, pH 5.5} \\
\hline$\alpha$-helix & 0.7 & 1.4 & 0 & 0 & 1.3 & 0.4 \\
\hline$\beta^{a}$ & 42.1 & 37.5 & 54.0 & 59.0 & 53.1 & 53.0 \\
\hline$\beta^{b}$ & 0 & 3.9 & 0 & 0 & 0.2 & 0 \\
\hline
\end{tabular}

\begin{tabular}{|c|c|c|c|c|c|c|}
\hline $\begin{array}{l}\text { Amylin } \\
\alpha \text {-helix }\end{array}$ & $\begin{array}{c}\text { QA in } \\
14.5\end{array}$ & $\begin{array}{c}\mathbf{n M} \text { so } \\
9.1\end{array}$ & $\begin{array}{l}\text { acet } \\
10.6\end{array}$ & $\begin{array}{c}\text { H } 5.5 \\
5.3\end{array}$ & 5.3 & 6.6 \\
\hline$\beta^{a}$ & 21.7 & 29.7 & 28.1 & 30.3 & 33.4 & 25.4 \\
\hline$\beta^{b}$ & 0 & 0 & 0 & 5.7 & 3.7 & 13.4 \\
\hline
\end{tabular}

${ }^{a}$ Antiparallel $\beta$-sheet. ${ }^{b}$ Parallel $\beta$-sheet. at $\approx 200 \mathrm{~nm}$ and a negative CD band at $\approx 225 \mathrm{~nm}$ in the presence of PMAQA indicates the formation of a predominant $\beta$ sheet structure (Fig. 2e). The observed CD minimum with a redshift (from $\approx 222$ on day 1 to $\approx 225 \mathrm{~nm}$ on day 5 ) in $\beta$-amyloid-140 correlates with previously observed $\beta$-sheet rich supramolecular structures in modified $\beta$-amyloid-1-40 and other small peptide aggregates. ${ }^{56,57}$

Next, we carried out FT-IR experiments on samples after 6 hours of incubation under similar experimental conditions used for CD measurements to further confirm the observations from CD experiments. As shown by the FT-IR spectra in Fig. 2g, predominant $\beta$-sheet structures of $\beta$-amyloid-1-40 were observed as indicated by a sharp amide I peak at $1628 \mathrm{~cm}^{-1}$ along with an increasing percentage of turns (at $1675 \mathrm{~cm}^{-1}$ ) in the presence of PMAQA, which are in qualitative agreement with CD results shown in Fig. 2e. In the case of amylin, FT-IR spectra showed a minor change in the characteristics of the helical band at $1650 \mathrm{~cm}^{-1}$ in the presence of PMAQA (Fig. 2h). This indicates that PMAQA binding restrains the conformation of partially folded amylin. ${ }^{43}$ The $\beta$-sheet rich $\beta$-amyloid and partially folded amylin conformations in the presence of PMAQA (Fig. 2) coupled with ThT observations (Fig. 1) indicated the presence of distinct peptide species such as fibers or loworder aggregates. To probe this, SEC profiling was performed to quantify the size distribution of the PMAQA bound peptide species. SEC analysis of $\beta$-amyloid-1-40 incubated with a 1.5 molar excess of PMAQA for $\sim 5$ minutes at room temperature showed two different elution profiles. The fractions collected at $\sim 5$ to $12 \mathrm{~mL}$ correspond to amyloid fibers or protofibers and those collected at $\sim 20$ to $25 \mathrm{~mL}$ are free polymers or low-order or monomeric $\beta$-amyloid-1-40 (Fig. S3a $\dagger$ ). ${ }^{58}$ Remarkably, the SEC profile for PMAQA-amylin mixed solution, incubated for a relatively longer time (overnight) at room temperature, exhibited low-order or monomeric amylin and free polymers eluted at $\approx 20$ to $25 \mathrm{~mL}$ (Fig. S3b†). ${ }^{59}$ Taken together, the above described experimental results present a counteractive role of PMAQA in $\beta$-amyloid-1-40 and amylin aggregation.

Next, we studied the binding mechanism of PMAQA with $\beta$ amyloid-1-40 or amylin using an integrated NMR and MD simulation approach. The amide-NH region of ${ }^{1} \mathrm{H}$ NMR spectra obtained in the absence of PMAQA showed monomer or loworder aggregates for both $\beta$-amyloid-1-40 and amylin as indicated by the dispersed NMR peaks (Fig. 3). A substantial change in the ${ }^{1} \mathrm{H}$ NMR spectra of $\beta$-amyloid-1-40 was observed at a $1: 0.02 \beta$-amyloid : PMAQA molar ratio (Fig. 3, pink trace). Remarkably, the loss of amide- ${ }^{1} \mathrm{H}$ peaks of $\beta$-amyloid-1-40 was observed when the PMAQA concentration $(1: 0.05 \beta$-amyloid : PMAQA ratio) was slightly increased (Fig. 4d). This agrees with the PMAQA induced $\beta$-amyloid-1-40 aggregation observed in ThT assays. In contrast, the amide peaks of amylin were observed even when titrated with a 1.2 molar equivalent of PMAQA (Fig. 3, blue trace). These NMR findings are in good agreement with the observed conformational transition from $\mathrm{CD}$ and ThT based aggregation results (Fig. $1 \mathrm{~b}$ and $2 \mathrm{~b}$ ). Interestingly, a significant line broadening was observed for the proton peak of the $-\mathrm{NR}_{3}{ }^{+}$group of PMAQA (at $2.97 \mathrm{ppm}$ ) in the presence of $\beta$-amyloid-1-40 which indicates the interaction of 


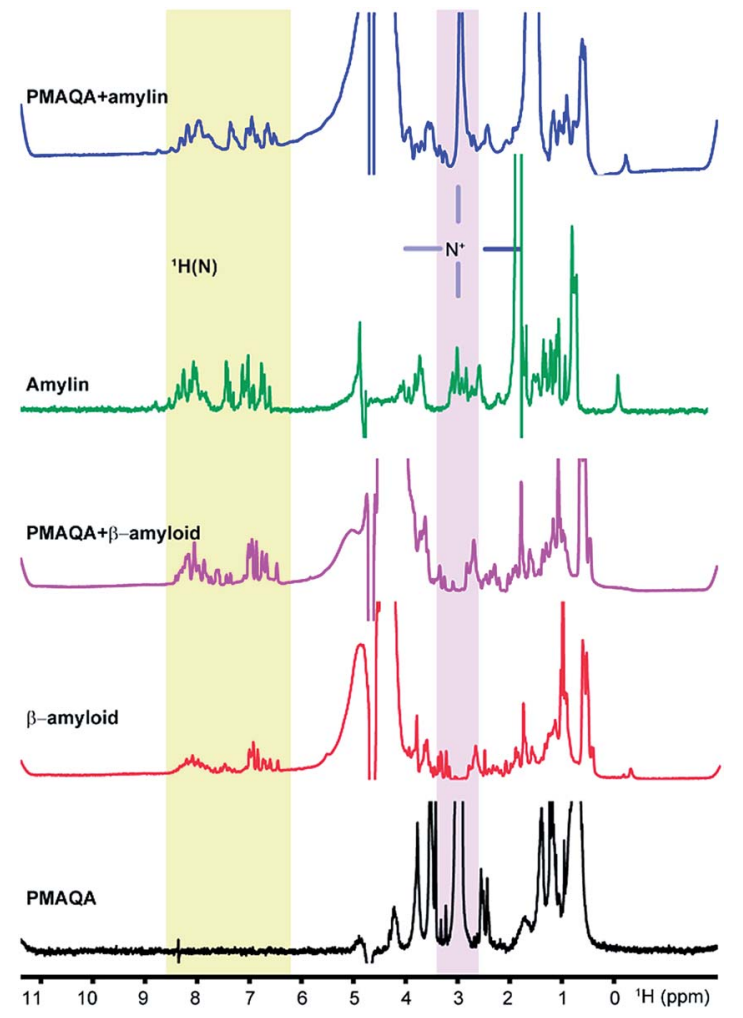

Fig. $3{ }^{1} \mathrm{H}$ NMR spectra of $\beta$-amyloid-1-40 or amylin. NMR spectra showing the interaction of PMAQA with $\beta$-amyloid-1-40 (60 $\mu \mathrm{M} \beta$ amyloid-1-40, 1.2 $\mu \mathrm{M}$ PMAQA dissolved in $10 \mathrm{mM}$ sodium phosphate, $\mathrm{pH}$ 7.4) or amylin (50 $\mu \mathrm{M}$ amylin, $60 \mu \mathrm{M}$ PMAQA dissolved in $30 \mathrm{mM}$ sodium acetate, $\mathrm{pH}$ 5.5). The change in the NMR signal intensity of the $-\mathrm{NR}_{3}{ }^{+}$proton in the PMAQA or protein amide region $(\mathrm{H}-\mathrm{N})$ is highlighted.

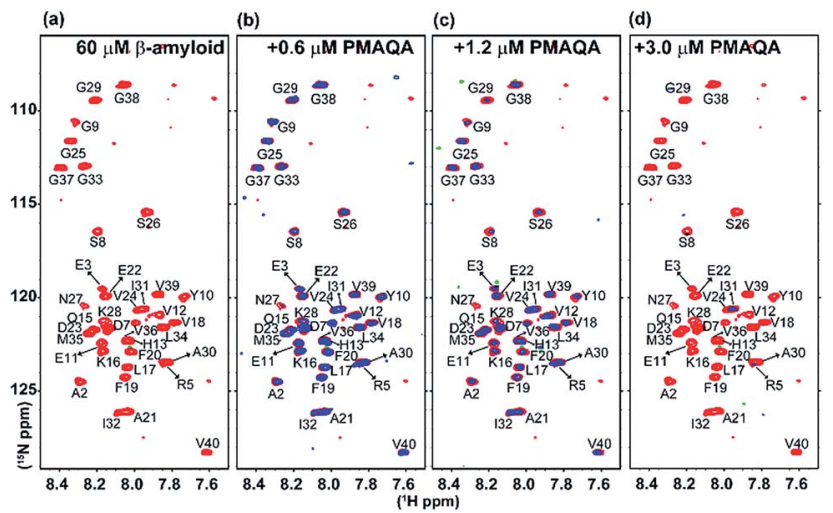

Fig. 4 SOFAST-HMQC NMR titration of PMAQA with $\beta$-amyloid-1-40. $2 \mathrm{D}{ }^{1} \mathrm{H} /{ }^{15} \mathrm{~N}$ SOFAST-HMQC NMR spectra of $\beta$-amyloid-1-40 $(60 \mu \mathrm{M})$ dissolved in $10 \mathrm{mM}$ sodium phosphate, $\mathrm{pH} 7.4$, (a) in the absence (red) or (b-d) presence of PMAQA (blue) at the indicated concentration (top). NMR spectra were recorded at $10^{\circ} \mathrm{C}$ on a $600 \mathrm{MHz}$ Bruker NMR spectrometer. Blue contours are presented with 1.5 times higher values as compared to red contours (in Sparky).

$-\mathrm{NR}_{3}{ }^{+}$with $\beta$-amyloid-1-40. In contrast, a sharp proton peak for $-\mathrm{NR}_{3}{ }^{+}$of PMAQA was observed in amylin solution (Fig. 3). This observation most likely indicates the formation of an electrostatic interaction between the cationic PMAQA and anionic $\beta$-amyloid-1-40, as $\beta$-amyloid-1-40 (at $\mathrm{pH}=7.4$ ) and amylin (at $\mathrm{pH}=5.5)$ carry negative $(-3)$ and positive $(+4)$ charges, respectively. ${ }^{\mathbf{6 0 , 6 1}}$ Thus, while the cationic group of PMAQA binds strongly to anionic $\beta$-amyloid-1-40, a repulsive force could be expected in the presence of cationic amylin.

To gain further atomistic insight into the mechanism, $\beta$ amyloid-1-40 aggregation was monitored using a $2 \mathrm{D}{ }^{15} \mathrm{~N} /{ }^{1} \mathrm{H}$ SOFAST-HMQC experiment at variable substoichiometric polymer concentrations; since ${ }^{1} \mathrm{H}$ NMR of amylin showed very little change in the amide region at superstoichiometric PMAQA concentrations, further NMR experiments were not carried out on amylin. As shown in Fig. 4a-d, intensity reduction and chemical shift changes were observed for $\beta$-amyloid-1-40 residues indicating a PMAQA induced structural rearrangement for $\beta$-amyloid-1-40. A substantial loss of signal intensity was observed in $\beta$-amyloid-1-40 spanning regions K16-V24 and A30V40 (as per previously published assignments ${ }^{62}$ ) indicates potential PMAQA binding sites on $\beta$-amyloid-1-40 at $1: 0.01$ and $1: 0.02 \beta$-amyloid : PMAQA molar ratios (Fig. $4 \mathrm{~b}$ and $\mathrm{c}$ ). A further increase in PMAQA concentration (1:0.05 $\beta$-amyloid : PMAQA molar ratio) increased the line-broadening and also resulted in the loss of ${ }^{15} \mathrm{~N} /{ }^{1} \mathrm{H}$ resonances (Fig. 4d). This indicates that PMAQA interaction with $\beta$-amyloid-1-40 induces the aggregation of the peptide.

To further explore the binding mechanism of PMAQA with amylin or $\beta$-amyloid-1-40 at the structural level, we performed all-atom MD simulation on a time scale of 0.7 or $1 \mu \mathrm{s}$, respectively. Structural analysis showed the formation of a substantial number of hydrogen bonds between PMAQA and $\beta$-amyloid-140 over a time-scale of $1 \mu$ s (Fig. S4a $\dagger$ ). All-atom MD simulation revealed potential electrostatic interactions or hydrogen bonds between the Glu22 and Asp23 residues of $\beta$-amyloid-1-40 and $-\mathrm{NR}_{3}{ }^{+}$of the polymer (Fig. 5a), whereas no interaction with the charged residues of $\beta$-amyloid-1-40 in the N-terminal (Asp1, Glu3 and Asp7) was observed. Other residues such as Leu17, Phe20, Val24, Ser26, Asn27, Ile31, and Ile32 were observed to interact with PMAQA through hydrogen bonding or hydrophobic interactions (Fig. 5a and Table S1†). This correlates with the NMR results that showed a substantial decrease in the NMR signal intensity in $\beta$-amyloid-1-40 regions spanning K16-V24 and A30-V40 (Fig. 5c). MD calculations also revealed several intermolecular hydrogen bonds and hydrophobic interactions between amylin and PMAQA (Fig. S4a $\dagger$ ). Amylin residues such as Asn21, Ile26, Ser28, Thr30, Asn31, Ser34 and Tyr37 were identified to be involved in intermolecular hydrogen bonding interactions with PMAQA (Fig. 5b and Table S2 $\dagger$ ). Overall, PMAQA exhibited a greater number of hydrogen bonds with amylin than with $\beta$-amyloid-1-40 indicating a relatively stronger binding affinity of PMAQA to amylin (Fig. S4a†).

Computation of binding energy using MM/PBSA presented distinct energetic parameters that favor PMAQA binding to $\beta$ amyloid-1-40 or amylin. The binding energy estimated for PMAQA- $\beta$-amyloid and PMAQA-amylin complexes was $-16.5 \pm$ 10.1 and $-27.4 \pm 11.7 \mathrm{kcal} \mathrm{mol}^{-1}$, respectively. The energetic parameter analysis shown in Table 2 indicates that coulombic and van der Waals interactions play a crucial role in PMAQA 
interaction between PMAQA $\left(-\mathrm{NR}_{3}{ }^{+}\right)$and Glu22/Asp23 in $\beta$ amyloid-1-40 could interfere with the early oligomer morphology and distribution of large $\beta$-amyloid-1-40 aggregates as observed in SEC (Fig. S3†). ${ }^{69}$ The binding of PMAQA to Glu22 and Val24 also affects the hydrophobic interaction between Val24 and Lys28 and electrostatic interaction between Lys28 and Glu22/Asp23. ${ }^{70}$

We next monitored the aggregation kinetics of amylin in real time using HS-AFM as the results presented above indicated an antagonistic behavior of PMAQA in amylin aggregation (Fig. 6). The fibrillation kinetics of freshly prepared amylin monomers was accelerated by adding preformed sonicated amylin amyloid seeds as described in the Experimental section. As shown in Fig. 6a (top trace), the addition of amylin monomers to preincubated amylin amyloid seeds (at $t=0 \mathrm{~s}$ ) induced a steady growth of fibers (Movie S1†). The seeding reaction (or fibril growth) and de novo nucleation (binding of additional fibril seeds to the stage) were observed (Fig. 6a). At 1000 s, we observed substantial growth in the fiber morphology; de novo nucleated amylin fibers and several fibers stopped growing after encountering other fibers at their growing end (Fig. 6a and Movie S1 $\dagger$ ). The growth of the selected amylin fiber (Fig. 6a, rectangle) in the absence of PMAQA is represented as kymographs as a function of time in one direction. The kymograph showed a substantial growth on the free end of the selected amylin fiber (Fig. 6b). These observations agree with the reported observations for other amyloidogenic peptides including $\beta$-amyloid. ${ }^{71,72}$ Remarkably, in the presence of equimolar PMAQA, both amylin fibril growth and de novo nucleation were found to be blocked (Fig. 6c and Movie S2 $\dagger$ ). In addition, no significant difference in the fiber length and population was
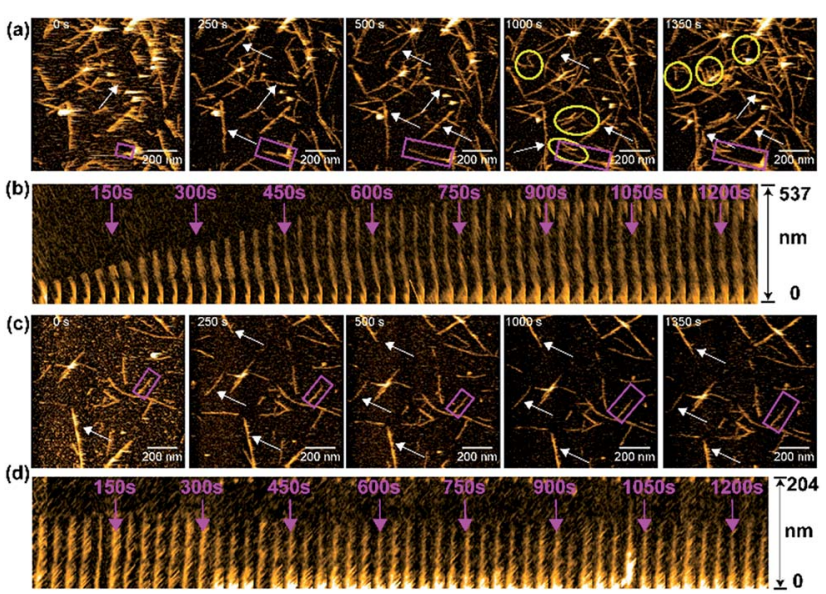

Fig. 6 Real-time monitoring of amylin fibrillation using HS-AFM. HSAFM images show the seeding reaction (fibril growth) of $5 \mu \mathrm{M}$ amylin monomers in the absence (a) or presence (c) of equimolar PMAQA. These images were extracted at the indicated time intervals from movies S1 and S2 (included in the ESI $†$ ). The growth of fibers as a function of time is indicated by arrows. The de novo nucleated amylin fibrils in (a) are shown inside the yellow circles. The kymographs for an individual selected fiber (shown inside pink rectangles in a and c) are shown for amylin in the absence (b) and presence of PMAQA (d). The kymographs show the growth of amylin fibers as a function of time (indicated with pink arrows) in the absence of PMAQA, whereas PMAQA blocks amylin nucleation and seeding processes. The scale bar is $200 \mathrm{~nm}$. observed between $0 \mathrm{~s}$ and $1350 \mathrm{~s}$ (Fig. 6c). Kymographs of amylin fibrillation in the presence of PMAQA showed no fiber growth. This indicates that PMAQA blocks the recruitment of amylin monomers to the growing ends of amyloid seeds that is needed to proceed the seeding reaction (Fig. 6d). These distinct morphological features observed for both bare mica and APTESmica suggest that the electrostatic interaction between the sample and the stage did not affect amylin aggregation and interactions with PMAQA. The HS-AFM experimental results, therefore, revealed the inhibitory activity of PMAQA towards
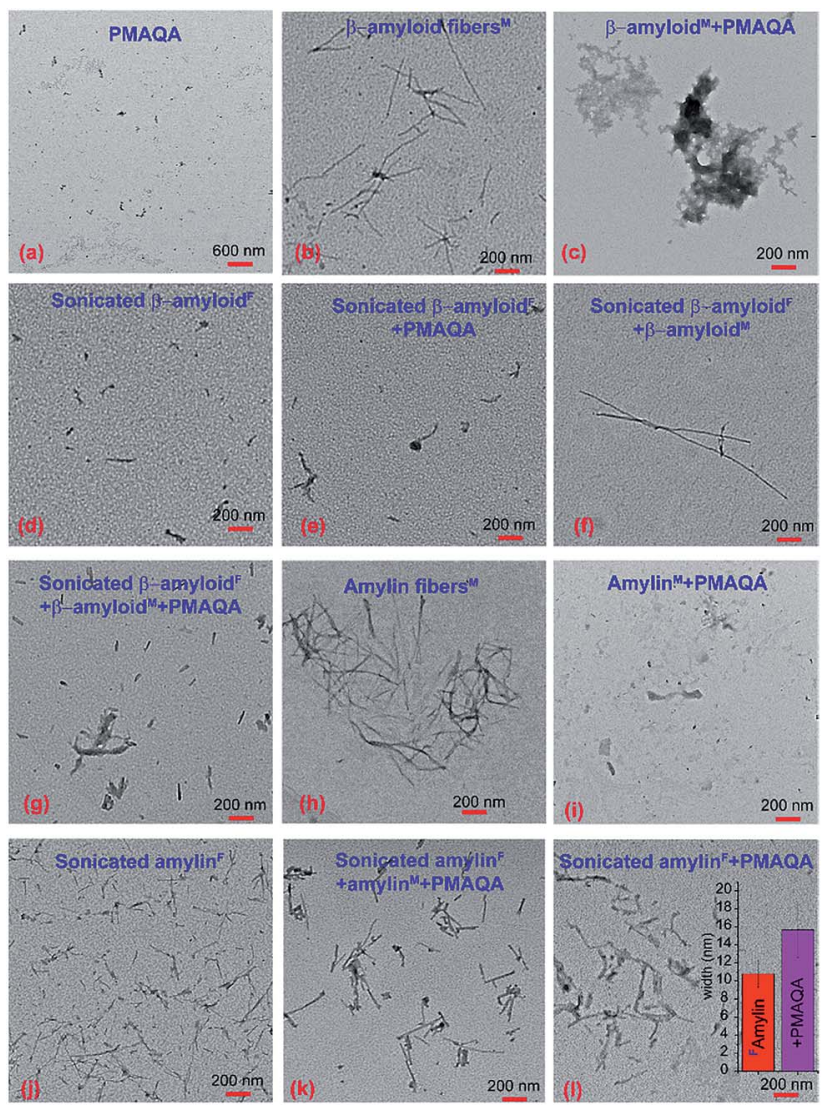

Fig. 7 TEM images of $\beta$-amyloid-1-40 and amylin interacting with PMAQA. TEM images of $5 \mu \mathrm{M}$ PMAQA (a) and $5 \mu \mathrm{M} \beta$-amyloid-1-40 in the absence (b) or presence (c) of an equivalent concentration of PMAQA incubated for 24 hours at room temperature under continuous agitation. TEM images of sonicated $\beta$-amyloid-1-40 fibers (d) prepared from matured fibers (see the Experimental section) in the presence of $5 \mu \mathrm{M}$ PMAQA (e); $5 \mu \mathrm{M} \beta$-amyloid-1-40 monomers (f); and $5 \mu \mathrm{M}$ PMAQA and $5 \mu \mathrm{M} \beta$-amyloid-1-40 monomers (g) incubated for 24 hours at room temperature under continuous agitation. TEM images of $5 \mu \mathrm{M}$ amylin in the absence (h) or presence (i) of $5 \mu \mathrm{M}$ PMAQA incubated for 24 hours at room temperature under continuous agitation. TEM images of sonicated amylin fibers (j) prepared from matured fibers (see the Experimental section) in the presence of $5 \mu \mathrm{M}$ PMAQA and $5 \mu \mathrm{M}$ amylin monomers (k) and $5 \mu \mathrm{M}$ PMAQA (l) incubated for 24 hours at room temperature under continuous agitation. The superscripts " $M$ " and " $F$ " denote the initial species of the peptide as monomers and fibers, respectively, used for the sample preparation. The inset in ( $I$ ) shows the average width of amylin fibers that were calculated from 10 distinct short amylin fibers selected randomly using the ImageJ plugin from (j) and (l). 
amylin aggregation at a molecular-level, which is consistent with other experimental results reported in this study.

We further characterized the morphology of $\beta$-amyloid-1-40 and amylin species present under various sample conditions (see the Experimental section). The TEM image of $5 \mu \mathrm{M}$ PMAQA showed very small particles (Fig. 7a), whereas freshly dissolved $\beta$-amyloid-1-40 monomers ( $5 \mu \mathrm{M})$ after continuous agitation at room temperature for 24 hours showed a fibril morphology (Fig. 7b). On the other hand, $\beta$-amyloid-1-40 monomers $(5 \mu \mathrm{M})$ incubated with an equimolar concentration of PMAQA exhibited amorphous-like aggregates (Fig. 7c). This correlates with the observed ThT fluorescence results that showed a substantial reduction in fluorescence intensity (Fig. 1a). We next examined the effect of PMAQA on $\beta$-amyloid-1-40 aggregation in the presence of amyloid seeds. TEM confirmed the formation of short fibrils when sonicating matured fibers for 2 hours (Fig. 7d). We did not observe a significant change in the sonicated fiber morphology incubated with equimolar PMAQA for 24 hours in the absence of peptide monomers (Fig. 7e). However, a remarkable difference in the $\beta$-amyloid-1-40 morphology was observed when a self-seeding reaction was performed in the absence or presence of PMAQA (Fig. 7f and g). The self-seeding reaction presented amyloid fiber growth in the absence of PMAQA (Fig. 7f), whereas in the presence of PMAQA short fibers and an amorphous-like fiber morphology were identified (Fig. 7g). Unlike $\beta$-amyloid-1-40, amylin monomers (5 $\mu \mathrm{M})$ incubated with equimolar PMAQA exhibited no fibers (Fig. 7i) which correlates with the ThT and CD results (Fig. 1b and 2b). Moreover, sonicated amylin fibers incubated with $5 \mu \mathrm{M}$ PMAQA for 24 hours in the absence of monomers showed a relatively thick fibril morphology as compared to the shortsized sonicated fibers (Fig. 7j-l). Interestingly, sonicated amylin fibers in the presence of equimolar peptide monomers and PMAQA showed no fibril growth which correlates with the HSAFM observations (Fig. 7k). A quantitative analysis of the widths of sonicated fibers (Fig. 7j) using the ImageJ plugin presented an average fiber width of $\sim 10.79 \mathrm{~nm}$. In the presence of PMAQA, the sonicated fibers depicted an average width of $\sim 15.69 \mathrm{~nm}$ indicating the possibility of PMAQA binding (Fig. 7l). Overall, the TEM analysis showed the formation of distinct morphological species of $\beta$-amyloid-1-40 and amylin in the presence or absence of PMAQA. These results further highlighted the opposite behaviours of PMAQA in the aggregation of $\beta$-amyloid-1-40 and amylin as observed in other biophysical experiments.

\section{Conclusions}

In conclusion, we have demonstrated the counter-activities of a cationic PMAQA polymer towards two different amyloidogenic peptides that are linked to $\mathrm{AD}$ and T2D. At substoichiometric concentrations, PMAQA showed significant inhibitory activity in amylin aggregation, whereas it significantly accelerated $\beta$ amyloid-1-40 aggregation by quickly altering the equilibrium state of $\beta$-amyloid-1-40 from an unfolded structure to a $\beta$-sheet structure. Our mechanistic study provides insights into the binding of PMAQA to $\beta$-amyloid-1-40 or amylin at the atomic level that could be helpful in understanding the modulation of peptide self-assembly and could aid in potential inhibitor designing. We believe that the opposite aggregation kinetics of the two different amyloidogenic proteins in the presence of a cationic polymer delineated in this study are likely to open avenues to test their potential therapeutic activities against an array of amyloid proteins involved in other human amyloid diseases by controlling functionalization of the chemical properties of the polymer.

\section{Conflicts of interest}

There are no conflicts to declare.

\section{Acknowledgements}

This study was supported by funds from NIH (AG048934 to A.R.). This work was (in part) performed under the International Collaborative Research Program of Institute for Protein Research, Osaka University, ICR-18-02.

\section{References}

1 T. P. J. Knowles, M. Vendruscolo and C. M. Dobson, Nat. Rev. Mol. Cell Biol., 2014, 15, 496.

2 S. A. Kotler, P. Walsh, J. R. Brender and A. Ramamoorthy, Chem. Soc. Rev., 2014, 43, 6692-6700.

3 C. A. Ross and M. A. Poirier, Nat. Med., 2004, 10, S10-S17.

4 L. M. Young, A. E. Ashcroft and S. E. Radford, Curr. Opin. Chem. Biol., 2017, 39, 90-99.

5 J. C. Sacchettini and J. W. Kelly, Nat. Rev. Drug Discovery, 2002, 1, 267-275.

6 J. Luo, S. K. T. S. Wärmländer, A. Gräslund and J. P. Abrahams, J. Biol. Chem., 2016, 291, 714576jbc.R116.

7 A. Abedini, A. Plesner, P. Cao, Z. Ridgway, J. Zhang, L. H. Tu, C. T. Middleton, B. Chao, D. J. Sartori, F. Meng, H. Wang, A. G. Wong, M. T. Zanni, C. B. Verchere, D. P. Raleigh and A. M. Schmidt, eLife, 2016, 5, e12977.

8 A. Spanopoulou, L. Heidrich, H.-R. Chen, C. Frost, D. Hrle, E. Malideli, K. Hille, A. Grammatikopoulos, J. Bernhagen, M. Zacharias, G. Rammes and A. Kapurniotu, Angew. Chem., Int. Ed., 2018, 57, 14503-14508.

9 Z. Liu, A. Zhang, H. Sun, Y. Han, L. Kong and X. Wang, RSC $A d v .$, 2017, 7, 6046-6058.

10 S.-Y. Hung and W.-M. Fu, J. Biomed. Sci., 2017, 24, 47.

11 A. J. Doig, M. P. Del Castillo-Frias, O. Berthoumieu, B. Tarus, J. Nasica-Labouze, F. Sterpone, P. H. Nguyen, N. M. Hooper, P. Faller and P. Derreumaux, ACS Chem. Neurosci., 2017, 8, 1435-1437.

12 H. Kroth, A. Ansaloni, Y. Varisco, A. Jan, N. Sreenivasachary, N. Rezaei-Ghaleh, V. Giriens, S. Lohmann, M. P. LópezDeber, O. Adolfsson, M. Pihlgren, P. Paganetti, W. Froestl, L. Nagel-Steger, D. Willbold, T. Schrader, M. Zweckstetter, A. Pfeifer, H. A. Lashuel and A. Muhs, J. Biol. Chem., 2012, 287, 34786-34800.

13 A. Pithadia, J. R. Brender, C. A. Fierke and A. Ramamoorthy, J. Diabetes Res., 2016, 2016, 204632. 
14 A. J. Doig and P. Derreumaux, Curr. Opin. Struct. Biol., 2015, 30, 50-56.

15 A. Granja, I. Frias, A. R. Neves, M. Pinheiro and S. Reis, BioMed Res. Int., 2017, 2017, 5813793.

16 Q. Song, H. Song, J. Xu, J. Huang, M. Hu, X. Gu, J. Chen, G. Zheng, H. Chen and X. Gao, Mol. Pharm., 2016, 13, 3976-3987.

17 M. Robinson, B. Yasie Lee and Z. Leonenko, AIMS Mol. Sci., 2015, 2, 332-358.

18 M. Stefani and S. Rigacci, Int. J. Mol. Sci., 2013, 14, 1241112457.

19 J. Bieschke, M. Herbst, T. Wiglenda, R. P. Friedrich, A. Boeddrich, F. Schiele, D. Kleckers, J. M. Lopez Del Amo, B. A. Grüning, Q. Wang, M. R. Schmidt, R. Lurz, R. Anwyl, S. Schnoegl, M. Fändrich, R. F. Frank, B. Reif, S. Günther, D. M. Walsh and E. E. Wanker, Nat. Chem. Biol., 2012, 8, 93-101.

20 S. Derrick, R. A. Kerr, Y. Nam, S. B. Oh, H. J. Lee, K. G. Earnest, N. Suh, K. L. Peck, M. Ozbil, K. J. Korshavn, A. Ramamoorthy, R. Prabhakar, E. J. Merino, J. Shearer, J. Lee, B. T. Ruotolo and M. H. Lim, J. Am, Chem. Soc., 2015, 137, 14785-14797.

21 M. Sato, K. Murakami, M. Uno, Y. Nakagawa, S. Katayama, K. I. Akagi, Y. Masuda, K. Takegoshi and K. Irie, J. Biol. Chem., 2013, 288, 23212-23224.

22 J. Kang, S. J. C. Lee, J. S. Nam, H. J. Lee, M. G. Kang, K. J. Korshavn, H. T. Kim, J. Cho, A. Ramamoorthy, H. W. Rhee, T. H. Kwon and M. H. Lim, Chem.-Eur. J., 2017, 23, 1645-1653.

23 B. R. Sahoo, T. Genjo, M. Bekier II, S. N. Cox, A. Stoddard, M. Ivanova, K. Yasuhara, C. Fierke, Y. Wang and A. Ramamoorthy, Chem. Commun., 2018, 54, 12883-12886.

24 Y. Song, E. G. Moore, Y. Guo and J. S. Moore, J. Am. Chem. Soc., 2017, 139, 4298-4301.

25 E. H. Pilkington, M. Lai, X. Ge, W. J. Stanley, B. Wang, M. Wang, A. Kakinen, M.-A. Sani, M. R. Whittaker, E. N. Gurzov, F. Ding, J. F. Quinn, T. P. Davis and P. C. Ke, Biomacromolecules, 2017, 18, 4249-4260.

26 C. Cabaleiro-Lago, O. Szczepankiewicz and S. Linse, Langmuir, 2012, 28, 1852-1857.

27 B. R. Sahoo, T. Genjo, S. J. Cox, A. K. Stoddard, G. M. Anantharamaiah, C. Fierke and A. Ramamoorthy, $J$. Mol. Biol., 2018, 430, 4230-4244.

28 J. Luo, C. H. Yu, H. Yu, R. Borstnar, S. C. L. Kamerlin, A. Gräslund, J. P. Abrahams and S. K. T. S. Wärmländer, ACS Chem. Neurosci., 2013, 4, 454-462.

29 A. Assarsson, S. Linse and C. Cabaleiro-Lago, Langmuir, 2014, 30, 8812-8818.

30 Y. Li, M. Cao and Y. Wang, J. Phys. Chem. B, 2006, 110, 18040-18045.

31 K. Yasuhara, J. Arakida, T. Ravula, S. K. Ramadugu, B. Sahoo, J.-I. Kikuchi and A. Ramamoorthy, J. Am. Chem. Soc., 2017, 139, 18657-18663.

32 W. Wang, A. Shao, N. Zhang, J. Fang, J. J. Ruan and B. H. Ruan, Sci. Rep., 2017, 7, 1-10.

33 D. Paolino, A. Vero, D. Cosco, T. M. G. Pecora, S. Cianciolo, M. Fresta and R. Pignatello, Front. Pharmacol., 2016, 7, 1-9.
34 K. Garai, S. L. Crick, S. M. Mustafi and C. Frieden, Protein Expr. Purif., 2009, 66, 107-112.

35 M. Dasari, A. Espargaro, R. Sabate, J. M. Lopez Del Amo, U. Fink, G. Grelle, J. Bieschke, S. Ventura and B. Reif, ChemBioChem, 2011, 12, 407-423.

36 P. Schanda and B. Brutscher, J. Am. Chem. Soc., 2005, 127, 8014-8015.

37 T. D. Goddard and D. G. Kneller, Univ. California, San Fr., 2004, vol. 14, p. 15, https://www.cgl.ucsf.edu/home/sparky/.

38 T. Ando, N. Kodera, E. Takai, D. Maruyama, K. Saito and A. Toda, Proc. Natl. Acad. Sci. U. S. A., 2001, 98, 12468-12472.

39 T. Uchihashi, N. Kodera and T. Ando, Nat. Protoc., 2012, 7, 1193-1206.

40 T. Watanabe-Nakayama and K. Ono, Methods Mol. Biol., 2018, 1814, 201-212.

41 A. K. Malde, L. Zuo, M. Breeze, M. Stroet, D. Poger, P. C. Nair, C. Oostenbrink and A. E. Mark, J. Chem. Theory Comput., 2011, 7, 4026-4037.

42 S. Vivekanandan, J. R. Brender, S. Y. Lee and A. Ramamoorthy, Biochem. Biophys. Res. Commun., 2011, 411, 312-316.

43 D. C. Rodriguez Camargo, K. Tripsianes, K. Buday, A. Franko, C. Göbl, C. Hartlmüller, R. Sarkar, M. Aichler, G. Mettenleiter, M. Schulz, A. Böddrich, C. Erck, H. Martens, A. K. Walch, T. Madl, E. E. Wanker, M. Conrad, M. H. De Angelis and B. Reif, Sci. Rep., 2017, 7, 44041.

44 D. Van Der Spoel, E. Lindahl, B. Hess, G. Groenhof, A. E. Mark and H. J. C. Berendsen, J. Comput. Chem., 2005, 26, 1701-1718.

45 N. Schmid, A. P. Eichenberger, A. Choutko, S. Riniker, M. Winger, A. E. Mark and W. F. Van Gunsteren, Eur. Biophys. J., 2011, 40, 843-856.

46 W. Humphrey, A. Dalke and K. Schulten, J. Mol. Graphics, 1996, 14, 33-38.

47 P. A. Kollman, I. Massova, C. Reyes, B. Kuhn, S. Huo, L. Chong, M. Lee, T. Lee, Y. Duan, W. Wang, O. Donini, P. Cieplak, J. Srinivasan, D. A. Case and T. E. Cheatham, Acc. Chem. Res., 2000, 33, 889-897.

48 D. Spiliotopoulos, A. Spitaleri and G. Musco, PLoS One, 2012, 7, e46902.

49 B. R. Sahoo, J. Maharana, M. C. Patra, G. K. Bhoi, S. K. Lenka, P. K. Dubey, S. Goyal, B. Dehury and S. K. Pradhan, Colloids Surf., B, 2014, 121, 307-318.

50 B. R. Sahoo, J. Maharana, G. K. Bhoi, S. K. Lenka, M. C. Patra, M. R. Dikhit, P. K. Dubey, S. K. Pradhan and B. K. Behera, Mol. BioSyst., 2014, 10, 1104-1116.

51 Y. Yoshimura, Y. Lin, H. Yagi, Y.-H. Lee, H. Kitayama, K. Sakurai, M. So, H. Ogi, H. Naiki and Y. Goto, Proc. Natl. Acad. Sci. U. S. A., 2012, 109, 14446-14451.

52 A. Micsonai, F. Wien, L. Kernya, Y.-H. Lee, Y. Goto, M. Réfrégiers and J. Kardos, Proc. Natl. Acad. Sci. U. S. A., 2015, 112, E3095-E3103.

53 Q. Wang, N. Shah, J. Zhao, C. Wang, C. Zhao, L. Liu, L. Li, F. Zhou and J. Zheng, Phys. Chem. Chem. Phys., 2011, 13, 15200. 
54 K. Ono, M. M. Condron and D. B. Teplow, Proc. Natl. Acad. Sci. U. S. A., 2009, 106, 14745-14750.

55 A. B. Soriaga, S. Sangwan, R. MacDonald, M. R. Sawaya and D. Eisenberg, J. Phys. Chem. B, 2016, 120, 5810-5816.

56 V. Castelletto, I. W. Hamley, P. J. Harris, U. Olsson and N. Spencer, J. Phys. Chem. B, 2009, 113, 9978-9987.

57 T. Guterman, M. Kornreich, A. Stern, L. Adler-Abramovich, D. Porath, R. Beck, L. J. W. Shimon and E. Gazit, Nat. Commun., 2016, 7, 1-10.

58 T. J. Esparza, N. C. Wildburger, H. Jiang, M. Gangolli, N. J. Cairns, R. J. Bateman and D. L. Brody, Sci. Rep., 2016, 6, 1-16.

59 Y. Bram, A. Frydman-Marom, I. Yanai, S. Gilead, R. ShaltielKaryo, N. Amdursky and E. Gazit, Sci. Rep., 2014, 4, 1-9.

60 A. Assarsson, E. Hellstrand, C. Cabaleiro-Lago and S. Linse, ACS Chem. Neurosci., 2014, 5, 266-274.

61 P. J. Marek, V. Patsalo, D. F. Green and D. P. Raleigh, Biochemistry, 2012, 51, 8478-8490.

62 J. Miguel, U. Fink, M. Dasari, G. Grelle, E. E. Wanker, J. Bieschke and B. Reif, J. Mol. Biol., 2012, 421, 517-524.

63 A. Gladytz, B. Abel and H. J. Risselada, Angew. Chem., Int. Ed., 2016, 55, 11242-11246.
64 M. Wang, A. Kakinen, E. H. Pilkington, T. P. Davis and P. C. Ke, Biomater. Sci., 2017, 5, 485-493.

65 B. K. Yoo, Y. Xiao, D. McElheny and Y. Ishii, J. Am. Chem. Soc., 2018, 140, 2781-2784.

66 Y. Miller, B. Ma and R. Nussinov, Proc. Natl. Acad. Sci. U. S. A., 2010, 107, 9490-9495.

67 V. S. Mithu, B. Sarkar, D. Bhowmik, M. Chandrakesan, S. Maiti and P. K. Madhu, Biophys. J., 2011, 101, 2825-2832.

68 S. Chakraborty and P. Das, Sci. Rep., 2017, 7, 1-12.

69 M. Guo, P. M. Gorman, M. Rico, A. Chakrabartty and D. V. Laurents, FEBS Lett., 2005, 579, 3574-3578.

70 M. A. Grant, N. D. Lazo, A. Lomakin, M. M. Condron, H. Arai, G. Yamin, A. C. Rigby and D. B. Teplow, Proc. Natl. Acad. Sci. U. S. A., 2007, 104, 16522-16527.

71 T. Watanabe-Nakayama, K. Ono, M. Itami, R. Takahashi, D. B. Teplow and M. Yamada, Proc. Natl. Acad. Sci. U. S. A., 2016, 113, 5835-5840.

72 P. E. Milhiet, D. Yamamoto, O. Berthoumieu, P. Dosset, C. le Grimellec, J. M. Verdier, S. Marchal and T. Ando, PLoS One, 2010, 5, e13240. 